\section{Review: early feeding after major gynaecological surgery is safe except for an increase in nausea}

\section{QUESTION}

In women having major abdominal gynaecological surgery, does early initiation of oral intake of food and fluids increase postoperative complications more than delayed initiation?

\section{METHODS}

Data sources: Cochrane Menstrual Disorders and Subfertility Group's specialised register of controlled trials, Medline, CINAHL, EMBASE/Excerpta Medica, and Cochrane Central Register of Controlled Trials (to April 2007); reference lists; and experts.

Study selection and assessment: randomised controlled trials (RCTs) that compared early $(<24 \mathrm{~h})$ with delayed ( $>24 \mathrm{~h}$, after resolution of postoperative ileus) initiation of oral intake of food and fluids after major open gynaecological surgery. Excluded were trials that were quasi-randomised or had no clear random allocation criteria; had important violations of the randomisation procedure or exclusions after allocation; or involved laparoscopic surgery or vaginal hysterectomy. 3 RCTs ( $\mathrm{n}=413$, mean age 41-57 y) of women with both malignant and benign disease met the selection criteria. Quality of individual trials was assessed based on criteria including allocation concealment, intention-to-treat analysis, blinding, and follow-up.
Outcomes: symptoms of postoperative ileus (nausea, vomiting, and abdominal distension); time to presence of bowel sounds, passage of flatus, passage of stool, and start of solid diet; and length of hospital stay.

\section{MAIN RESULTS}

Early feeding increased risk of nausea, but groups did not differ for vomiting, abdominal distension, or ileus (table). Early feeding reduced time to bowel sounds by 0.5 day $(95 \%$ CI 0.2 to $0.8 ; 1 \mathrm{RCT}, \mathrm{n}=195)$ and time to solid diet by 1.5 days (CI 0.7 to $2.3 ; 2$ RCTs, $n=301$ ). Groups did not differ for time to passage of flatus or stool, or length of hospital stay.

\section{CONCLUSIONS}

In women having major abdominal gynaecological surgery, early initiation of oral intake of food and fluids increases risk of nausea but not other postoperative complications. Early feeding results in faster resumption of some aspects of bowel function and earlier return to a solid diet.

\section{ABSTRACTED FROM}

Charoenkwan K, Phillipson G, Vutyavanich T. Early versus delayed (traditional) oral fluids and food for reducing complications after major abdominal gynaecologic surgery. Cochrane Database Syst Rev 2007;(4):CD004508.

Correspondence to: Dr K Charoenkwan, Chiang Mai University, Chiang Mai, Thailand kicharoe@mail.med.cmu.ac.th

Source of funding: not stated.

- Clinical impact ratings: Genitourinary surgery 5/7; Perioperative 6/7

Early $v$ delayed initiation of oral intake of food and fluids after major abdominal gynaecological surgery*

\begin{tabular}{|c|c|c|c|c|c|}
\hline \multirow[b]{2}{*}{ Outcomes } & \multirow[b]{2}{*}{ Number of trials (n) } & \multicolumn{2}{|c|}{ Weighted event rates } & \multirow[b]{2}{*}{ RRI (95\% Cl) } & \multirow[b]{2}{*}{ NNH (Cl) } \\
\hline & & Early feeding & Delayed feeding & & \\
\hline Nausea & $1(195)$ & $43 \%$ & $24 \%$ & $79 \%$ (19 to 171$)$ & 6 (4 to 17$)$ \\
\hline \multirow[t]{2}{*}{ Abdominal distension } & $2(301)$ & $34 \%$ & $31 \%$ & $7 \%(-23$ to 47$)$ & Not significant \\
\hline & & & & RRR (CI) & NNT \\
\hline Vomiting & $2(301)$ & $6.1 \%$ & $7.1 \%$ & $14 \%(-100$ to 63$)$ & Not significant \\
\hline
\end{tabular}

${ }^{*}$ Abbreviations defined in glossary. RRI, RRR, NNH, NNT, and Cl calculated from data in article using a fixed-effects model.

onsiderable attention has been paid to speedy resumption of oral nutrition after surgery. Evidence suggests that paralytic ileus after surgery is transient and has little clinical effect on the return to oral nutrition in the early postoperative phase. An important impetus for early return to oral nutrition is shorter hospital stay.

Charoenkwan et al conducted a comprehensive review of studies that assessed outcomes after early postoperative feeding in women having gynaecological surgery; only 3 trials met the inclusion criteria. The authors acknowledged the variability in the quality of the trials and the limited data available for individual outcomes. Data for some major morbidity outcomes (ie, nausea, postoperative ileus, nasogastric tube placement, and febrile morbidity) were provided by only 1 study each. Outcomes related to patient preference, cost, and physiological benefits of early postoperative feeding were lacking; these areas present a clear opportunity for future research.

The schedule for early return to oral nutrition varied slightly among the included studies. One study offered patients a soft diet on the first postoperative day and a solid diet on the second day, whereas the other 2 studies offered clear fluids on the first postoperative day, advancing to a solid diet as tolerated.

Although oral nutrition can be initiated safely in patients having major gynaecological surgery, nausea is often a discomforting factor. Therefore, decisions about early feeding should be made jointly by nurses, physicians, and patients and should reflect the patient's ability and desire to ingest nutrition.

\section{Salima SJ Ladak, RN, MN}

University Health Network, Toronto General Hospital Toronto, Ontario, Canada 